\title{
Liouvillian Solutions of Irreducible Second Order Linear Difference Equations
}

\author{
Mark van Hoeij* \\ Department of Mathematics \\ Florida State University \\ Tallahassee, FL 32306, USA \\ hoeij@math.fsu.edu
}

\author{
Giles Levy \\ Department of Mathematics \\ Florida State University \\ Tallahassee, FL 32306, USA \\ glevy@math.fsu.edu
}

\begin{abstract}
In this paper we give a new algorithm to compute Liouvillian solutions of linear difference equations. The first algorithm for this was given by Hendriks in 1998, and Hendriks and Singer in 1999. Several improvements have been published, including a paper by Cha and van Hoeij that reduces the combinatorial problem. But the number of combinations still depended exponentially on the number of singularities. For irreducible second order equations, we give a short and very efficient algorithm; the number of combinations is 1 .
\end{abstract}

\section{Categories and Subject Descriptors}

G.2.1 [Combinatorics]: [Recurrences and difference equations]; I.1.2 [Symbolic and Algebraic Manipulation]: Algorithms- $A l$ gebraic algorithms

\section{General Terms}

Algorithms

\section{INTRODUCTION}

Let $\tau$ denote the shift operator: $\tau(f(n))=f(n+1)$. An operator $L_{1}=\tau^{2}+a_{1} \tau+a_{0}$ with $a_{0}, a_{1} \in \mathbb{C}(n)$ corresponds to a recurrence relation $u(n+2)+a_{1}(n) u(n+1)+a_{0}(n) u(n)=0$. Algorithms for finding rational resp. hypergeometric resp. Liouvillian solutions have been given in [2, 4] resp. [7, 5, 6] resp. [11, 12, 3, 14]. The algorithms for hypergeometric solutions use a combinatorial search, where each of the combinations involves computing polynomial solutions. The algorithms for Liouvillian solutions are also combinatorial in nature, either because they call an algorithm for hypergeometric solutions $[11,12,3]$, or perform a reduced (but still exponential) combinatorial search [14].

A second order operator $L_{1}$ is irreducible if and only if it does not have hypergeometric solutions. If $L_{1}$ is irreducible, then the task of finding a gauge transformation (see Definition 8) from $L_{1}$ to an operator of the form $L_{2}=\tau^{2}+b_{0}$ with $b_{0} \in \mathbb{C}(n)$ is equivalent

${ }^{*}$ Supported by NSF grant 0728853

Permission to make digital or hard copies of all or part of this work for personal or classroom use is granted without fee provided that copies are not made or distributed for profit or commercial advantage and that copies bear this notice and the full citation on the first page. To copy otherwise, to republish, to post on servers or to redistribute to lists, requires prior specific permission and/or a fee.

ISSAC 2010, 25-28 July 2010, Munich, Germany.

Copyright 2010 ACM 978-1-4503-0150-3/10/0007 ...\$5.00. to finding Liouvillian solutions of $L_{1}$ ([12, Lemma 4.1]). Finding such a transformation is useful because $\tau^{2}+b_{0}$ is easily solved with interlaced hypergeometric terms as follows:

Let the factorization of $b_{0}$ into monic linear factors be $c \frac{p_{1} p_{2} \cdots p_{i}}{q_{1} q_{2} \cdots q_{j}}, c \in$ $\mathbb{C}$, then $\tau^{2}+b_{0}$ has solutions

$$
\frac{\Gamma\left(p_{1} / 2\right) \Gamma\left(p_{2} / 2\right) \cdots \Gamma\left(p_{i} / 2\right)}{\Gamma\left(q_{1} / 2\right) \Gamma\left(q_{2} / 2\right) \cdots \Gamma\left(q_{j} / 2\right)} \cdot c^{n / 2} \cdot\left\{\begin{array}{l}
k_{1}, \text { if } n \text { even } \\
k_{2}, \text { if } n \text { odd }
\end{array}\right.
$$

where $k_{1}, k_{2}$ are arbitrary constants.

As an example we look at $A 099364$ from 'The On-Line Encyclopedia of Integer Sequences' or OEIS ([1]): A099364 is a sequence named "An inverse Chebyshev transform of $(1-x)^{2}$ " and satisfies $(n+6) u(n+2)+2 u(n+1)-(8+4 n) u(n)=0$. Our implementation ([16] or [15]) returns the solution:

$$
u(n)=\left(\frac{1}{6} n+\frac{5}{6}\right) v(n)-\left(\frac{1}{12} n+\frac{1}{2}\right) v(n+1)
$$

$$
\text { where } v(n+2)-\frac{4(n+2)}{n+7} v(n)=0 \text { and }\{v(0)=0, v(1)=-2\}
$$

so $v(n)$ can be represented as in equation (1). Prior algorithms for Liouvillian solutions will also solve this equation, using a search that depends exponentially on the number of finite singularities (see [14] for more details). This paper introduces a new method for finding Liouvillian solutions. It works for irreducible linear difference equations of order 2. Our algorithm is short and easy to implement [16], but its primary benefit is that it is very efficient because Corollary 1 allows us to reduce the number of combinations to 1 .

The idea behind the algorithm is as follows (for notations see Section 2). Suppose that $L_{1}=\tau^{2}+a_{1} \tau+a_{0}$ and $L_{2}=\tau^{2}+b_{0}$ with $b_{0}, a_{0}, a_{1} \neq 0$, and $b_{0}$ unknown. If there exists a gauge transformation $G: L_{1} \rightarrow L_{2}$ then there is an induced transformation between their symmetric squares $L_{1}^{\Theta 2}, L_{2}^{\Theta 2}$, which are of order 3 and 2 respectively. The induced mapping must therefore have a one dimensional kernel, which corresponds to a hypergeometric solution of $L_{1}^{\circledR 2}$. But we can find this hypergeometric solution without a combinatorial search, because Corollary 1 reduces the problem to computing a rational solution.

\section{PRELIMINARIES}

More detailed preliminaries can be found in [15].

Definition 1. $\tau$ will refer to the shift operator acting on $\mathbb{C}(n)$ and $\operatorname{Mat}_{a \times b}(\mathbb{C}(n))$ by $\tau: n \mapsto n+1$.

An operator $L=\sum_{i} a_{i} \tau^{i}$ acts as $L u(n)=\sum_{i} a_{i} u(n+i)$. 
DefinItion 2. $\mathbb{C}(n)[\tau]$ is the ring of linear difference operators where ring multiplication is composition of operators $L_{1} L_{2}=L_{1}$ 。 $L_{2}$, e.g. $(\tau-a(n))(\tau-b(n))=\tau^{2}-(a(n)+b(n+1)) \tau+a(n) b(n)$.

We let $\tau$ operate on $u(n) \in \mathbb{C}^{\mathbb{N}}$ by $u(n) \mapsto u(n+1)$.

Definition 3. ['Galois Theory of Difference Equations' Example 1.3 [10]] Let $S=\mathbb{C}^{\mathbb{N}} / \sim$ where $s_{1} \sim s_{2}$ if there exists $N \in \mathbb{N}$ such that, for all $n>N, s_{1}(n)=s_{2}(n)$.

The reason for using $S$ is that the dimension of the solution space will be equal to the order of the difference operator (see Theorem 1 below). Working in $S$ also enables us to work in $\mathbb{C}[n][\tau]$ as well as in $\mathbb{C}(n)[\tau]$. In particular, if $L \in \mathbb{C}(n)[\tau]$ and we multiply away the denominators of the coefficients to obtain an element of $\mathbb{C}[n][\tau]$ then the solution space does not change when working in $S$.

Definition 4. $V(L)$ refers to the solution space of the operator $L$, i.e. $V(L)=\{u \in S \mid L u=0\}$, where $S$ is as in Definition 3.

Example 1. For $L=\tau+n+1$ we write $V(L)=\mathbb{C} \cdot(-1)^{n} \Gamma(n+1)$ or $V(L)=\mathbb{C} \cdot[1,-1,2,-6,24,-120, \ldots]$.

Definition 5. A unit is a sequence in $S$ that is invertible, i.e. a sequence that only has finitely many zeros.

THEOREM 1. [' $A=B$ ' Theorem 8.2.1 [9]] Let $L=\sum_{k=0}^{r} a_{k} \tau^{k}$ be a linear difference operator of order $r$ on $S$. If $a_{r}$ and $a_{0}$ are units, then $\operatorname{dim}(\operatorname{ker}(L))=r$.

We can view $\mathbb{C}(n)$ as a subset of $S$ so the theorem applies to $L \in \mathbb{C}(n)[\tau]$ with $a_{0}, a_{r} \neq 0$.

Definition 6. A function or sequence $v(n)$ for which $v(n+1) / v(n)$ is a rational function of $n$ will be called a hypergeometric term. Such $a v(n)$ will be called a hypergeometric solution of any $L \in$ $\mathbb{C}(n)[\tau]$ for which $L v=0$. It corresponds to a first order right hand factor of $L$, namely $\tau-r(n)$ where $r(n)=v(n+1) / v(n)$.

A hypergeometric function is a function $\sum_{n=0}^{\infty} v(n) x^{n}$ where $v(n)$ is a hypergeometric term.

\subsection{Gauge Transformations}

Let $D=\mathbb{C}(n)[\tau]$. If $L \in D \backslash\{0\}$ then $D / D L$ is a $D$-module.

Definition 7. $L_{1}$ is gauge equivalent to $L_{2}$ when $D / D L_{1}$ and $D / D L_{2}$ are isomorphic as $D-$ modules.

Lemma 1. $L_{1}$ is gauge equivalent to $L_{2}$ if and only if there exists $G \in D$ such that $G\left(V\left(L_{1}\right)\right)=V\left(L_{2}\right)$ and $L_{1}, L_{2}$ have the same order. Thus $G$ defines a bijection $V\left(L_{1}\right) \rightarrow V\left(L_{2}\right)$.

Note: If $D / D L_{1} \cong D / D L_{2}$ then $G$ in the Lemma corresponds to the image in $D / D L_{1}$ of the element $1 \in D / D L_{2}$.

Definition 8. The bijection defined by G in Lemma 1 above will be called a gauge transformation.

Definition 9. Let $r(n)=c p_{1}(n)^{e_{1}} \cdots p_{j}(n)^{e_{j}} \in C(n)$ with $C \subseteq$ C. Let the $e_{i} \in \mathbb{Z}$, let the $p_{i}(n)$ be irreducible in $C[n]$, and let $s_{i} \in C$ be the sum of the roots of $p_{i}(n)$. Then $r(n)$ is said to be in shift normal form if $-\operatorname{deg}\left(p_{i}(n)\right)<\operatorname{Re}\left(s_{i}\right) \leqslant 0$, for $i=1, \ldots, j$. We denote $\operatorname{SNF}(r(n))$ as the shift normalized form of $r(n)$ which is obtained by replacing each $p_{i}(n)$ by $p_{i}\left(n+k_{i}\right)$ for some $k_{i} \in \mathbb{Z}$ such that $p_{i}\left(n+k_{i}\right)$ is in shift normal form. Two rational functions, $r_{1}(n), r_{2}(n)$ will be called shift equivalent, denoted $r_{1}(n) \stackrel{\text { SE }}{\equiv} r_{2}(n)$, if $\operatorname{SNF}\left(r_{1}(n)\right)=\operatorname{SNF}\left(r_{2}(n)\right)$
REMARK 1. Let $r_{1}, r_{2} \in \mathbb{C}(n)$. If $r_{1}(n) \stackrel{\text { SE }}{\equiv} r_{2}(n)$ then there exists $r \in \mathbb{C}(n)$ such that $r_{1} / r_{2}=r(n+1) / r(n)$. This is easy to prove if $r_{1}, r_{2}$ are irreducible polynomials. The general case reduces to the irreducible case because the relation $\stackrel{\mathrm{SE}}{\equiv}$ is closed under multiplication, and the same is true for the group of all $r(n+1) / r(n)$.

Definition 10. The companion matrix of a monic difference operator

$$
L=\tau^{k}+a_{k-1} \tau^{k-1}+\cdots+a_{0}, a_{i} \in \mathbb{C}(n)
$$

which is satisfied by $u(n)$ will refer to the matrix:

$$
M=\left(\begin{array}{ccccc}
0 & 1 & \ldots & 0 & 0 \\
\vdots & \vdots & \ddots & \vdots & \vdots \\
0 & 0 & \ldots & 1 & 0 \\
0 & 0 & \ldots & 0 & 1 \\
-a_{0} & -a_{1} & \ldots & -a_{k-2} & -a_{k-1}
\end{array}\right)
$$

The equation $L u=0$ is equivalent to the system $\tau(Y)=M Y$ where

$$
Y=\left(\begin{array}{c}
u(n) \\
\vdots \\
u(n+k-1)
\end{array}\right) .
$$

Definition 11. Let $L=a_{k} \tau^{k}+a_{k-1} \tau^{k-1}+\cdots+a_{0}, a_{i} \in \mathbb{C}(n)$. The determinant of $L, \operatorname{det}(L):=(-1)^{k} a_{0} / a_{k}$, i.e. the determinant of its companion matrix.

Lemma 2. If there exists a gauge transformation $G: V\left(L_{1}\right) \rightarrow$ $V\left(L_{2}\right)$ then $\operatorname{det}\left(L_{1}\right) \stackrel{\text { SE }}{\equiv} \operatorname{det}\left(L_{2}\right)$.

The proof follows by examining the matrix representations of $L_{1}$, $L_{2}$, and $G$. If $\hat{G}$ is the matrix representation of $G$ then $\operatorname{det}\left(L_{2}\right)=$ $\operatorname{det}\left(L_{1}\right) \tau(\operatorname{det}(\hat{G})) / \operatorname{det}(\hat{G})$, for details see [15].

\subsection{Liouvillian Solutions and Symmetric Prod- ucts}

Definition 12 (Definition 6 in [6]). Let $L_{1}, L_{2} \in \mathbb{C}(n)[\tau]$. The symmetric product (called term product in [15]) $L_{1} \otimes L_{2}$ of $L_{1}$ and $L_{2}$ is defined as the monic operator $L \in \mathbb{C}(n)[\tau]$ of smallest order such that $L\left(u_{1} u_{2}\right)=0$ for all $u_{1}, u_{2} \in S$ with $L_{1} u_{1}=0$ and $L_{2} u_{2}=0$.

The following is an example of the symmetric product of a second order $L_{1}=\tau^{2}+b(n) \tau+c(n)$ with a first order $L_{2}=\tau-a(n)$ :

$$
L_{1} \otimes L_{2}=\tau^{2}+b(n) a(n+1) \tau+c(n) a(n) a(n+1) .
$$

Definition 13. The symmetric square of $L$, denoted $L^{\circledR 2}$, will refer to the symmetric product of $L$ and $L$.

Liouvillian solutions are defined in [12] Section 3.2. For irreducible operators they are characterized by the following theorem.

THeorem 2. (Propositions 31-32 in [8], or Lemma 4.1 in [12]): An irreducible $k$ 'th order operator $L$ has Liouvillian solutions if and only if its companion matrix is gauge equivalent to one that can be written as

$$
M=\left(\begin{array}{ccccc}
0 & 1 & \ldots & 0 & 0 \\
\vdots & \vdots & \ddots & \vdots & \vdots \\
0 & 0 & \ldots & 0 & 1 \\
-a & 0 & \ldots & 0 & 0
\end{array}\right), a \in \mathbb{C}(n) .
$$

In other words, $L$ is gauge equivalent to $\tau^{k}+a$. 
Lemma 3. Let $L=a_{2} \tau^{2}+a_{1} \tau+a_{0}, a_{i} \in \mathbb{C}[n], a_{0}, a_{2} \neq 0$.

1. If $a_{1} \neq 0$ then

$$
L^{\circledR 2}=c_{3} \tau^{3}+c_{2} \tau^{2}+c_{1} \tau+c_{0},
$$

where:

$$
\begin{aligned}
& c_{3}=a_{1}(n) a_{2}(n+1)^{2} a_{2}(n) \\
& c_{2}=a_{1}(n+1) a_{2}(n)\left(-a_{1}(n+1) a_{1}(n)+a_{0}(n+1) a_{2}(n)\right) \\
& c_{1}=a_{0}(n+1) a_{1}(n)\left(a_{1}(n+1) a_{1}(n)-a_{0}(n+1) a_{2}(n)\right) \\
& c_{0}=-a_{1}(n+1) a_{0}(n+1) a_{0}(n)^{2} .
\end{aligned}
$$

If $a_{1}=0$ then

$$
L^{(2)}=a_{2}^{2} \tau^{2}-a_{0}^{2} .
$$

2. $L^{\circledR 2}$ has order: $\left\{\begin{array}{l}2, \text { if } a_{1}=0 \\ 3, \text { if } a_{1} \neq 0\end{array}\right.$

For a proof of the lemma, which relies on the linear independence of the solutions of $L$, see [15].

REMARK 1. The proof of the Lemma below illustrates computations in Step 4 of Algorithm FindLiouvillian in Section 3.

LemMA 4. Let $a \neq 0$. Given a gauge transformation from $L=$ $\tau^{2}+a(n) \tau+b(n)$ to $\hat{L}=\tau^{2}+r(n)$ one can compute a difference operator mapping $V\left(L^{\circledR 2}\right)$ onto $V\left(\hat{L}^{\circledR 2}\right)$.

Proof. Let $u(n) \in V(L)$ and $v(n) \in V(\hat{L})$ with $v(n)=g_{0}(n) u(n)+$ $g_{1}(n) u(n+1)$, then

$$
v(n)^{2}=g_{0}(n)^{2} u(n)^{2}+2 g_{0}(n) g_{1}(n) u(n) u(n+1)+g_{1}(n)^{2} u(n+1)^{2} \text {. }
$$

The substitution (obtained by squaring $u(n+2)=-a(n) u(n+1)-$ $b(n) u(n))$ :

$$
u(n) u(n+1)=\frac{u(n+2)^{2}-a(n)^{2} u(n+1)^{2}-b(n)^{2} u(n)^{2}}{2 a(n) b(n)}
$$

yields:

$$
\begin{gathered}
v(n)^{2}=\frac{g_{0}(n)\left(-g_{1}(n) b(n)+g_{0}(n) a(n)\right)}{a(n)} u(n)^{2} \\
-\frac{g_{1}(n)\left(-g_{1}(n) b(n)+g_{0}(n) a(n)\right)}{b(n)} u(n+1)^{2}+\frac{g_{0}(n) g_{1}(n)}{a(n) b(n)} u(n+2)^{2} .
\end{gathered}
$$

Since $V\left(L^{\circledR 2}\right)$ is spanned by squares, by linear extension equation (6) will define a map from $V\left(L^{\circledR 2}\right)$ to $V\left(\hat{L}^{\circledR 2}\right)$. To prove that this map is onto, choose linearly independent $u_{1}, u_{2} \in V(L)$. Applying the gauge transformation $g_{0}(n) \tau^{0}+g_{1}(n) \tau$ produces linearly independent $v_{1}, v_{2} \in V(\hat{L})$. Then $v_{1}^{2}, v_{2}^{2}$ must be linearly independent as well, and hence form a basis of $V\left(\hat{L}^{\circledR 2}\right)$. This basis is the image of $u_{1}^{2}, u_{2}^{2}$ under the map given by (6), and hence this map is onto (so the kernel must then have dimension 3-2 = 1)

Lemma 5. With notations as in Lemma 4, if L is irreducible then

\section{1. $\hat{L}$ is irreducible}

\section{2. $\hat{L}^{\circledR 2}$ is irreducible}

Proof. The first item follows from Definitions 7, 8 and the assumption that $L$ is irreducible. For the second item, assume that $\tau-s$ is a right-hand factor of $\hat{L}^{\circledR 2}=\tau^{2}-r^{2}$. Then $r^{2}=s \tau(s)$. Suppose that $s$ is not a square, then take a point $p \in \mathbb{C}$ with maximal real part, for which $s$ has a root or pole at $p$ of odd order. Then $s \tau(s)$ has a root or pole at $p$ with odd order, contradicting $r^{2}=s \tau(s)$. Hence $s$ is a square in $\mathbb{C}(n)$, say $s=t^{2}$. So $r= \pm t \tau(t)$, and after possibly multiplying $s$ by $\sqrt{-1}$ we have $r=-t \tau(t)$. But then $\tau-t$ is a right-hand factor of $\hat{L}=\tau^{2}+r$, contradicting item 1 .

\section{ALGORITHM FINDLIOUVILLIAN}

For explanations and comments on the steps in the algorithm, see Section 4.

Algorithm FindLiouvillian

Input: $L \in \mathbb{C}[n][\tau]$ a second order, irreducible, homogeneous difference operator. Write $L=a_{2}(n) \tau^{2}+a_{1}(n) \tau+a_{0}(n)$.

Output: A two-term difference operator, $\hat{L}$, with a gauge transformation from $\hat{L}$ to $L$, if it exists.

1. If $a_{1}=0$ then return $\hat{L}=L$ and stop.

2. Let $u(n)$ be an indeterminate function. Impose the relation $L u(n)=0$, i.e.

$$
u(n+2)=-\frac{1}{a_{2}(n)}\left(a_{0}(n) u(n)+a_{1}(n) u(n+1)\right) .
$$

3. Let $d=\operatorname{det}(L)=a_{0} / a_{2}$. Let $R$ be a non-zero rational solution of

$$
L_{T}:=L^{\circledR 2} \otimes(\tau+1 / d),
$$

if such solution exists, else return NULL and stop. A formula for $L_{T}$ is $d(n+2) d(n+1) d(n) c_{3}(n) \tau^{3}-d(n+1) d(n) c_{2}(n) \tau^{2}+$ $d(n) c_{1}(n) \tau-c_{0}(n)$ where the $c_{i}$ are from (4).

4. Let $g$ be an indeterminate and let $v(n)=g u(n)+u(n+1)$. Compute $d_{0}, d_{1}, d_{2} \in \mathbb{C}(n)[g]$ such that

$$
v(n)^{2}=d_{0} u(n)^{2}+d_{1} u(n+1)^{2}+d_{2} u(n+2)^{2} .
$$

(To compute $d_{0}, d_{1}, d_{2}$ first substitute Equation (7) into Equation (8).)

5. Let $S$ denote a non-zero solution of $\tau+d$, so $\tau(S)=-d(n) S$ and $\tau^{2}(S)=d(n+1) d(n) S$. Substitute the following

$$
\begin{aligned}
u(n)^{2} & =R(n) S(n) \\
u(n+1)^{2} & =-R(n+1) d(n) S(n) \\
u(n+2)^{2} & =R(n+2) d(n+1) d(n) S(n)
\end{aligned}
$$

into Equation (8) to get $v(n)^{2}=S(n) A$ for some $A \in \mathbb{C}(n)[g]$.

6. Solve $A=0$ for $g$ and choose one solution. $A$ is a quadratic equation so this solution is in $\mathbb{C}(n)$ or in a quadratic extension of $\mathbb{C}(n)$. If $g \notin \mathbb{C}(n)$ then return an error message and stop.

7. Return $\hat{L}$ as well as the transformation $V(\hat{L}) \rightarrow V(L)$ :

$$
\hat{L}=\tau^{2}+b(n) \frac{\tau(\delta)}{\delta}
$$

$$
u(n)=\frac{1}{\delta}((g(n+1)-a(n)) v(n)-v(n+1))
$$

where $a(n)=a_{1} / a_{2}, b(n)=a_{0} / a_{2}, \delta=g(n) g(n+1)-$ $g(n) a(n)+b(n)$, and $v(n)$ denotes a solution of $\hat{L}$.

REMARK 2. The formula for the gauge transformation given in Step 7 was found by computing the inverse of the gauge transformation $v(n)=g u(n)+u(n+1)$ introduced in Step 4 (where the value of $g$ is computed in Steps 5 and 6).

Example 2. Consider $n u(n+2)-u(n+1)-\left(n^{2}-1\right)(2 n-1) u(n)=$ 0. By downloading FindLiouvillian [16] and running it on $n \tau^{2}-$ 
$\tau-\left(n^{2}-1\right)(2 n-1)$ one obtains:

$$
\begin{aligned}
d= & -\left(n^{2}-1\right)(2 n-1) / n \\
L_{T}= & n(n+3)(2 n+3)(n+1)^{2} \tau^{3}- \\
& n(n+2)\left(2 n^{3}+3 n^{2}-n+1\right) \tau^{2}- \\
& (n+2)(n+1)\left(2 n^{3}+3 n^{2}-n+1\right) \tau+ \\
& n(n+2)(n-1)(n+1)(2 n-1)
\end{aligned}
$$

(denominators were multiplied away by taking the primitive part)

$$
\begin{gathered}
R=\frac{1}{n}, \quad A=\frac{1}{n} \cdot\left(g^{2}+(3 n-2) g+(2 n-1)(n-1)\right) \\
g=1-n, \quad \delta=1-n^{2}
\end{gathered}
$$

and finally:

$$
\hat{L}=\tau^{2}-(2 n-1)(n+2), \quad u(n)=\frac{1}{n} v(n)+\frac{1}{n^{2}-1} v(n+1) .
$$

The algorithm is explained in Section 4 below. As mentioned in the Introduction, the main point is to show that the hypergeometric solution of $L^{\circledR 2}$ corresponds to a rational solution of $L^{\circledR 2} \otimes(\tau+$ $1 / \operatorname{det}(L))$ as this is what allows us to eliminate the combinatorial aspect of the algorithm.

\section{EXPLANATION}

Idea: Assume that $L=\tau^{2}+a_{1} \tau+a_{0}, a_{1} \neq 0$ is Liouvillian and irreducible, then there exists a gauge transformation $L \rightarrow \hat{L}$ for some $\hat{L}$ of the form $\tau^{2}+r$ (see Theorem 2). $\operatorname{dim}\left(V\left(L^{\circledR 2}\right)\right)=3$ and $\operatorname{dim}\left(V\left(\hat{L}^{\circledR 2}\right)\right)=2$ so the transformation $L^{\circledR 2} \rightarrow \hat{L}^{\circledR 2}$ has a 1 -dimensional kernel corresponding to a right hand factor of $L^{\circledR 2}$ (i.e. $L^{\circledR 2}$ has a hypergeometric solution, namely $R S$ in Step 5). This gives us the gauge transformation from $V(L)$ to $V(\hat{L})$. In order that Step 3 only needs to search for a solution $\in \mathbb{C}(n)$ (which is easier than computing a more general hypergeometric solution) we work with the symmetric product of $L^{\Theta 2}$ and $\tau-1 / \operatorname{det}(L)$ and use Theorem 3 .

\subsection{Algorithm Step 3}

Let $L$ be from Input and let $\hat{L}$ be a two-term operator.

Remark 2. A symmetric product of a second order two-term operator with a first order difference operator is again a second order two-term operator. It follows that we may disregard the symmetric product, i.e. we need only search for a gauge transformation.

Lemma 6. Let $A_{1}, A_{2}, G_{2} \in \mathbb{C}(n)[\tau], \operatorname{ord}\left(A_{1}\right)=3, \operatorname{ord}\left(A_{2}\right)=2$, and assume that $G_{2}\left(V\left(A_{1}\right)\right)=V\left(A_{2}\right)$, i.e. $V\left(A_{1}\right) \stackrel{G_{2}}{\rightarrow} V\left(A_{2}\right)$. Then $A_{1}$ has a first order right hand factor, as well a a second order left hand factor that is gauge equivalent to $A_{2}$.

Proof. $V\left(\operatorname{GCRD}\left(A_{1}, G_{2}\right)\right)=V\left(A_{1}\right) \cap V\left(G_{2}\right)=\operatorname{ker}\left(G_{2}: V\left(A_{1}\right) \rightarrow\right.$ $\left.V\left(A_{2}\right)\right)$ which has dimension $3-2=1$ and so $A_{1}$ has a first order right hand factor, $L_{1}=\operatorname{GCRD}\left(A_{1}, G_{2}\right)$. Write $A_{1}=L_{2} L_{1}, G_{2}=$ $\tilde{G} L_{1}$ then $\tilde{G}: V\left(L_{2}\right) \rightarrow V\left(A_{2}\right)$ shows that $L_{2}$ is gauge equivalent to $A_{2}$.

For the theorem below we now substitute $L^{\circledR 2}, \hat{L}^{\circledR 2}$ for $A_{1}, A_{2}$, respectively, from the preceding Lemma.

THEOREM 3. Let $\hat{L}=r_{2} \tau^{2}+r_{0}$ and let $L=a_{2} \tau^{2}+a_{1} \tau+a_{0}, a_{i} \neq 0$. Suppose there is a gauge transformation $G: V(L) \rightarrow V(\hat{L})$ then by Lemma 4 there is $G_{2}: V\left(L^{\circledR 2}\right) \rightarrow V\left(\hat{L}^{\Theta 2}\right)$. Let $L_{1}=\operatorname{GCRD}\left(G_{2}, L^{\circledR 2}\right)$ (which has order 1 by Lemma 6 ) and write $L^{\circledR 2}=L_{2} L_{1}$. Then

$$
\operatorname{det}\left(L_{1}\right) \stackrel{\text { SE }}{\equiv}-\operatorname{det}(L) .
$$

Proof. $\quad 1 . \operatorname{det}(L) \stackrel{\text { SE }}{=} \operatorname{det}(\hat{L})$, see Lemma 2 .

2. $\operatorname{det}\left(L^{\circledR 2}\right) \stackrel{\text { SE }}{\equiv} \operatorname{det}(L)^{3}$, see the formula for $L^{\circledR 2}$ in Lemma 3 .

3. $\operatorname{det}\left(\hat{L}^{\circledR 2}\right)=-\operatorname{det}(\hat{L})^{2} \stackrel{\text { SE }}{\equiv}-\operatorname{det}(L)^{2}$, see Lemma 3 with $a_{1}=0$ and Item 1 , respectively.

4. $\operatorname{det}\left(L^{(2)}\right)=\operatorname{det}\left(L_{2} L_{1}\right) \stackrel{\text { SE }}{\equiv} \operatorname{det}\left(L_{2}\right) \operatorname{det}\left(L_{1}\right)$.

5. $L_{2}$ is gauge equivalent to $\hat{L}^{\circledR 2}$, see Lemma 6 .

6. $\operatorname{det}\left(L_{2}\right) \stackrel{\mathrm{SE}}{\equiv} \operatorname{det}\left(\hat{L}^{\Theta 2}\right)=-\operatorname{det}(\hat{L})^{2} \stackrel{\mathrm{SE}}{\equiv}-\operatorname{det}(L)^{2}$, see Items $5,3,1$.

7. $\operatorname{det}\left(L_{1}\right) \stackrel{\text { SE }}{\equiv}-\operatorname{det}(L)$, see Items $4,2,6$.

Corollary 1. Let $L, \hat{L}, G$ be as in Theorem 3 so that $L^{\circledR 2}=$ $L_{2} L_{1}$ then there exists a rational solution of $L^{\circledR 2} \otimes(\tau+1 / \operatorname{det}(L))$.

Step 3 computes this rational solution. The solution is rational because the first order factor has determinant shift equivalent to 1 (the solution is $r(n)$ from Remark 1). Lemma 5 combined with Lemma 6 shows that if $L$ is irreducible, then $L_{2}$ in the corollary is irreducible, which implies that the rational solution is unique (up to constant multiples).

\subsection{Algorithm Step 4}

Lemma 7. If $T_{1}: g_{1} \tau+g_{0}$ with $g_{1} \neq 0$ defines a gauge transformation from $L$ to a two-term operator then $T_{2}: \tau+g_{0} / g_{1}$ is also a gauge transformation from $L$ to a two-term operator.

The two transformations differ by the term product $u \mapsto g_{1} u$ and so the Lemma's claim follows from Remark 2. (The case $g_{1}=0$ defines a term product.)

\subsection{Algorithm Step 5}

Equation (8) defines the map $L^{\circledR 2} \rightarrow \hat{L}^{\circledR 2}$ and $R S$ is in the kernel. Both $u(n)^{2}$ and $R S$ satisfy $L^{\circledR 2}$, see steps 2 and 3. (Recall that $R S$ is the hypergeometric solution of $L^{\circledR 2}$ that we computed using Corollary 1.)

\subsection{Algorithm Step 6}

It can be proven that if there exists a gauge transformation from $L \in \mathbb{C}(n)[\tau]$ to an operator of the form $\tau^{2}+\alpha$ where $\alpha$ is algebraic over $\mathbb{C}(n)$ then there also exists a gauge transformation $G: L \rightarrow \tilde{L}=$ $\tau^{2}+\tilde{\alpha}$ with $G, \tilde{L} \in \mathbb{C}(n)[\tau]$. Note that if $L \in C(n)[\tau], C \subset \mathbb{C}$ then an algebraic extension of $C$ may occur, see the following example.

ExAmple 3. $L_{1}=\tau^{2}-\tau-n^{2}+1$ and $L_{2}=\tau^{2}-(n+i)(n+1-i)$. are gauge equivalent with $L_{1} \in \mathbb{Q}(n)[\tau]$ and $L_{2} \in \mathbb{Q}(n)[\tau, \sqrt{-1}]$. Both $\frac{1}{n-i} \tau+1$, which sends $L_{2}$ to $L_{1}$, and its inverse are $\in \mathbb{C}(n)[\tau]$.

\section{ADDITIONAL WORK}

It is interesting to note that if we introduce a minus sign in Step 3 of Algorithm FindLiouvillian, setting $d:=-a_{0} / a_{2}$, then the algorithm turns into the so-called eigenring method (Section 4 in [4]). Specifically, the $\tau+g$ computed in the algorithm (this time, use both solutions of $A$ ) will then be right-hand factors of $L$ (this is not a complete algorithm for factoring $L$, it works if $L$ has precisely two first order right-hand factors in $\mathbb{C}(n)[\tau])$.

To generalize our algorithm to $n$ 'th order, we need to compute a gauge transformation from $L$ to $L \otimes\left(\tau-\zeta_{n}\right)$ where $\zeta_{n}$ is an $n$ 'th root of unity. So we need to find or write an implementation that can compute gauge transformations while allowing algebraic numbers in the input. 


\section{REFERENCES}

[1] N. J. A. Sloane. The On-Line Encyclopedia of Integer Sequences. World-Wide Web URL www.research.att.com/ njas/sequences/

[2] S. A. Abramov. Rational solutions of linear difference and $q$-difference equations with polynomial coefficients. In (Russian) Programmirovanie, 6: 3-11, 1995; translation in Program. Comput. Software 21, 6: 273-278, (1995).

[3] S.A. Abramov, M.A. Barkatou, D.E. Khmelnov. On $m$-Interlacing Solutions of Linear Difference Equations, LNCS, 5743, p. 1-17 (2009).

[4] M. A. Barkatou. Rational Solutions of Matrix Difference Equations: The Problem of Equivalence and Factorization, ISSAC'1999, p. 277-282, (1999).

[5] M. van Hoeij. Finite singularities and hypergeometric solutions of linear recurrence equations. In Journal of Pure and Applied Algebra, 139: 109-131, 1999.

[6] T. Cluzeau, M. van Hoeij. Computing Hypergeometric Solutions of Linear Recurrence Equations. In Applicable Algebra in Engineering, Communication and Computing, 17(2): 83-115, 2006.

[7] M. Petkovšek. Hypergeometric solutions of linear recurrences with polynomial coefficients. In Journal of Symbolic Computation, 14(2-3): 243-264, 1992.

[8] R. Feng, M. F. Singer, M. Wu. Liouvillian solutions of difference-differential equations. To appear in Journal of Symbolic Computation, 2009.
[9] M. Petkovšek, H. S. Wilf, D. Zeilberger. $A=B$. With a foreword by Donald E. Knuth. A. K. Peters, Ltd., Wellesley, MA, 1996.

[10] M. van der Put, M. F. Singer. Galois theory of difference equations. In Lecture Notes in Mathematics, vol. 1666, Springer, Berlin, 1997.

[11] P.A. Hendriks An algorithm for determining the difference Galois group for second order linear difference equations. In Journal of Symbolic Computation, 26: 445âĂŞ462 1998.

[12] P. A. Hendriks, M. F. Singer. Solving difference equations in finite terms. In Journal of Symbolic Computation, 27: 239-259, 1999.

[13] M. F. Singer. Testing reducibility of linear differential operators: a group theoretic perspective. In Applicable Algebra in Engineering, Communication and Computing, 7(2): 77-104, 1996.

[14] Y. Cha, M. van Hoeij. Liouvillian solutions of irreducible linear difference equations. In ISSAC '09: Proceedings of the 2009 International Symposium on Symbolic and Algebraic Computation, Pages: 87-94, 2009.

[15] G. Levy. Solutions of second order recurrence relations. Ph.D. dissertation, Florida State University, 2010. Text and implementations available at: http://www.math.fsu.edu/ glevy/implementation

[16] M. van Hoeij, G. Levy. FindLiouvillian implementation. http://www.math.fsu.edu/ hoeij/files/FindLiouvillian 